\title{
A Novel Two-Beam Accelerator (Twobetron)
}

\author{
Y. Y. Lau, Ya. S. Derbenev, R. M. Gilgenbach, \\ J. W. Luginsland, J. M. Hochman, and M. T. Walter \\ Intense Energy Beam Interaction Laboratory \\ Department of Nuclear Engineering \\ University of Michigan \\ Ann Arbor, Michigan 48109-2104
}

\begin{abstract}
A new configuration is analyzed wherein a low current beam is accelerated to high energies (10's of amps, 10's of MeV) by a driver beam of high current and low energy (a few kiloamps, $<1 \mathrm{MeV})$. The annular driver beam excites the TM 020 cavity mode of an accelerating structure which transfers its if power to the on-axis secondary beam. Systematic variation of the driver beam radius provides the secondary beam with phase focusing and adjustable acceleration gradient. A proof-of-principle experiment is suggested. Various issues, such as the scaling laws, transverse and longitudinal instabilities, rf coupling among cavities, etc., are examined.
\end{abstract}

\section{INTRODUCTION}

Two-beam accelerators have been studied extensively in the high energy physics community [1]. This paper concentrates on the $10 \mathrm{MeV}$ range. Compact electron and ion accelerators in this energy range have a wide range of applications, such as treatment of bulk materials, activation analysis, and medical radiation sources. To achieve such an energy at moderate levels of current (tens of amps) requires considerable power, and a natural candidate for a driver is the pulse power system. Intense annular electron beams (multi-kiloamps, $<1 \mathrm{MeV}$ ) extracted from such a system have been modulated efficiently, and the current modulations exhibit a high degree of amplitude and phase stability [2]. Their successful applications [3] in ultra-high power microwave generation and in particle acceleration have motivated us to use them as drivers in a novel two-beam accelerator [4], termed "twobetron" hereafter.

The twobetron is shown schematically in Fig. 1. The driver beam is an annular beam of radius $r_{0}$, carrying an AC current $I_{d}$ at frequency $\omega$. It passes through an accelerator structure, consisting of $\mathrm{N}$ cylindrical pillbox cavities. Each cavity has a radius $b=5.52 \mathrm{c} / \omega$ so that $\omega$ is also the resonant frequency of the $\mathrm{TM}_{020}$ mode of the pillbox cavity [Fig. 1]. The secondary beam is an on-axis pencil beam, carrying an AC current $I_{S}\left(I_{S} \ll I_{d}\right)$, also at frequency $\omega$. Since the rf electric fields of the TM020 mode have opposite signs in the outer region and in the inner region, the mode retards the annular driver beam but accelerates the onaxis secondary beam. If the driver beam radius is modulated axially, phase focusing and tunability in the output energy of the secondary beam can be 
achieved. This phase focusing technique thus also offers the possibility of using much lower driver beam voltage (e.g., $100 \mathrm{keV}$ ), a distinct advantage in many applications.

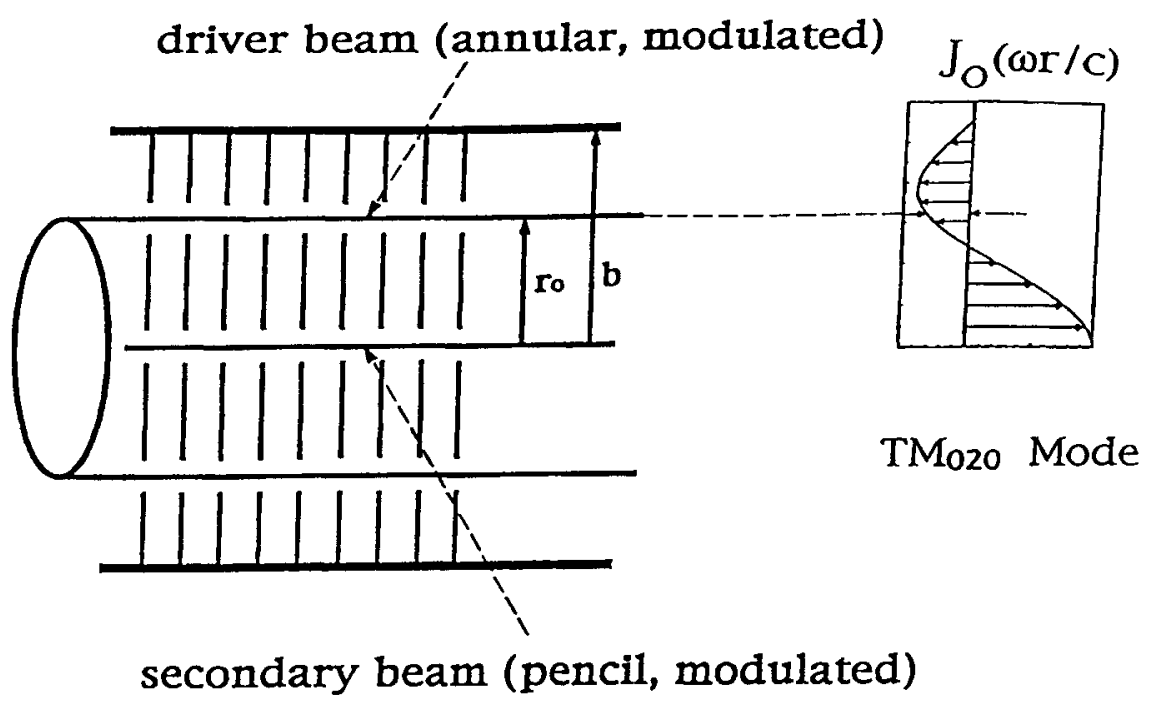

Figure 1. Schematic drawing of the two-beam accelerator. Also shown is the $\mathrm{rf}$ force profile, $\mathrm{J}_{\mathrm{O}}(\omega \mathrm{r} / \mathrm{c})$, associated with the axial electric field of the $\mathrm{TM}_{020}$ cavity mode.

\section{ACCELERATION MECHANISM}

To calculate the excitation of the $\mathrm{TM}_{020}$ mode by the primary beam, and the resultant acceleration of the secondary beam by this mode, we assume that the intense space charge on the beam does not alter the rf characteristic of the cavities. We also assume, for the time being, that the individual pillbox cavities are electromagnetically isolated from each other when the beams are absent. Since the cavities are excited mainly by the rf current $I_{d}$ carried by the primary beam, the $\mathrm{TM}_{020}$ mode so excited always decelerates the primary beam electrons on the average (by conservation of energy). This is true whether the beam radius $r_{o}$ is larger or smaller than a, where $a=2.405 \mathrm{c} / \omega$ is the radius of the rf electric field null of the $\mathrm{TM}_{020}$ mode [Fig. 2a]. The value of the rf electric field at $r_{0}$ then gives the deceleration gradient. In terms of the relativistic mass factor $\left(\gamma_{d}\right)$, the energy loss by this driver beam as it traverses the $n$-th cavity is given by 


$$
\frac{\mathrm{d} \gamma_{\mathrm{d}}}{\mathrm{dn}}=-\Lambda \delta^{2}
$$

in a continuum description. In Eq. (1),

$$
\Lambda=0.066(\omega \mathrm{L} / \mathrm{c}) \mathrm{Q}\left(\mathrm{I}_{\mathrm{d}} / 1 \mathrm{kA}\right)
$$

is the dimensionless parameter that measures the strength of the cavity excitation by the primary beam,

$$
\delta=\mathrm{J}_{\mathrm{o}}\left(\omega \mathrm{r}_{\mathrm{o}} / \mathrm{c}\right) \approx-1.249\left(\mathrm{r}_{\mathrm{o}}-\mathrm{a}\right) / \mathrm{a},
$$

$Q$ is the quality factor of the $T M_{020}$ mode, $L$ is the cavity length, $J_{O}$ is the Bessel function of the first kind of order zero. In writing the last expression of Eq. (3), we have made the assumption that the annular beam is located at the vicinity of the rf electric field-null $\left(r_{0} \approx a\right)$.

(A)
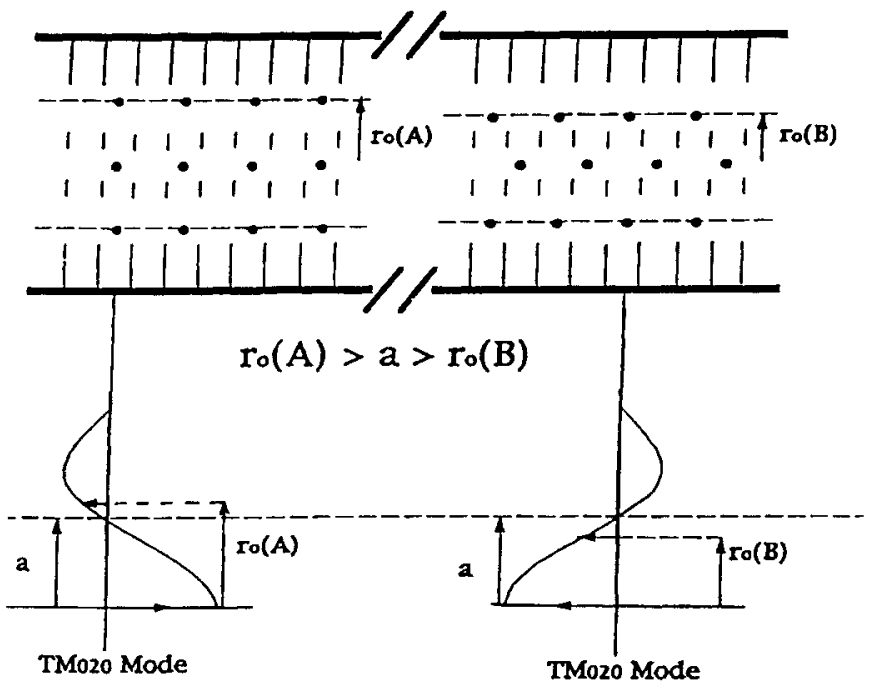

Figure 2.

(a) Left. Position of the primary beam radius $r_{0}\left(r_{0}>a\right)$ for secondary beam acceleration when both beams enter the cavity at the same phase.

(b) Right. Position of the primary beam radius $r_{0}\left(r_{0}<a\right)$ for secondary beam acceleration when both beams enter the cavity at $180^{\circ}$ apart phase. 
If the secondary beam enters the cavity at the same phase as the primary beam, the former will be accelerated if $r_{0}>a$, for in this case the rf fields experienced by both beams have opposite polarity [Fig. 2a]. Since the rf electric field has a radial dependence of $J_{0}(\omega r / c)$, it is obvious that $1 /|\delta|$ is the "transformer ratio", which is the ratio of the energy gain by the secondary beam to the energy loss by the primary beam, if both beams enter the cavity at the same phase. This dependence on the phase is reflected in the following equation which describes the change in the relativistic mass factor $\left(\gamma_{\mathrm{S}}\right)$ of the secondary beam as it traverses the $\mathrm{n}$-th cavity:

$$
\frac{\mathrm{d} \gamma_{\mathrm{S}}}{\mathrm{dn}}=-\Lambda \delta \cdot \cos \left(\theta_{\mathrm{s}}-\theta_{\mathrm{d}}\right)
$$

where $\theta_{\mathrm{S}}$ is the phase of the secondary beam bunch and $\theta_{\mathrm{d}}$ is the phase of the primary beam bunch when they enter the $n$-th cavity. Equation (4) is readily obtained from Eq. (1) by noting the transformer ratio $1 / \delta$ and the phase difference mentioned above. Equations (3) and (4) indeed show that $\gamma_{s}$ increases if $r_{0}>a$ and if $\theta_{\mathrm{d}}=\boldsymbol{\theta}_{\mathrm{s}}$.

The secondary beam cannot be accelerated indefinitely because of the increase in the phase slippage between $\theta_{\mathrm{d}}$ and $\theta_{\mathrm{S}}$ downstream. This phase slippage occurs as the primary beam is decelerated and the secondary beam is accelerated. Its rate of increase is governed by

$$
\frac{d\left(\theta_{s}-\theta_{d}\right)}{d n}=\frac{\omega L}{c}\left(\frac{1}{\beta_{s}}-\frac{1}{\beta_{d}}\right)=\frac{\omega L}{c}\left[\frac{1}{\sqrt{1-1 / \gamma_{s}^{2}}}-\frac{1}{\sqrt{1-1 / \gamma_{d}^{2}}}\right]
$$

The effect on the secondary beam by this phase slippage is illustrated in Fig. 3, which is obtained by numerically solving the system of three equations [(1), (4), (5)] in three unknowns: $\gamma_{d}, \gamma_{s}, \theta_{s}-\theta_{d}$. The initial conditions for these three unknowns are taken to be: $\theta_{\mathrm{s}}-\theta_{\mathrm{d}}=0, \gamma_{\mathrm{d}}=\gamma_{\mathrm{s}}=2.37$, corresponding to an initial energy of $700 \mathrm{keV}$ for both beams. The other parameters are : $\omega / 2 \pi=3.65$ $\mathrm{GHz}, \mathrm{b}=7.221 \mathrm{~cm}, \mathrm{~L}=1 \mathrm{~cm}, \mathrm{a}=3.146 \mathrm{~cm}, \mathrm{r}_{\mathrm{o}}=3.322 \mathrm{~cm}, \mathrm{Q}=100, \mathrm{I}_{\mathrm{d}}=0.5 \mathrm{kA}$. Since we have taken $L=1 \mathrm{~cm}$, the cavity number $n$ is also the axial distance $(z)$ in $\mathrm{cm}$.

Figure 3a shows that $\gamma_{\mathrm{d}}$ decreases from the initial value of 2.37 to 1.24 at $\mathrm{n}=90$, i.e., the primary beam's energy steadily decreases from $700 \mathrm{keV}$ to $125 \mathrm{keV}$ after propagating $90 \mathrm{~cm}$. The secondary beam's energy [Fig. 3b] increases initially, reaching a maximum value of $2.3 \mathrm{MeV}$ after $24 \mathrm{~cm}$, and then decreases 
due to the phase slippage until $n=56$, and oscillates further downstream as the phase slippage continues.
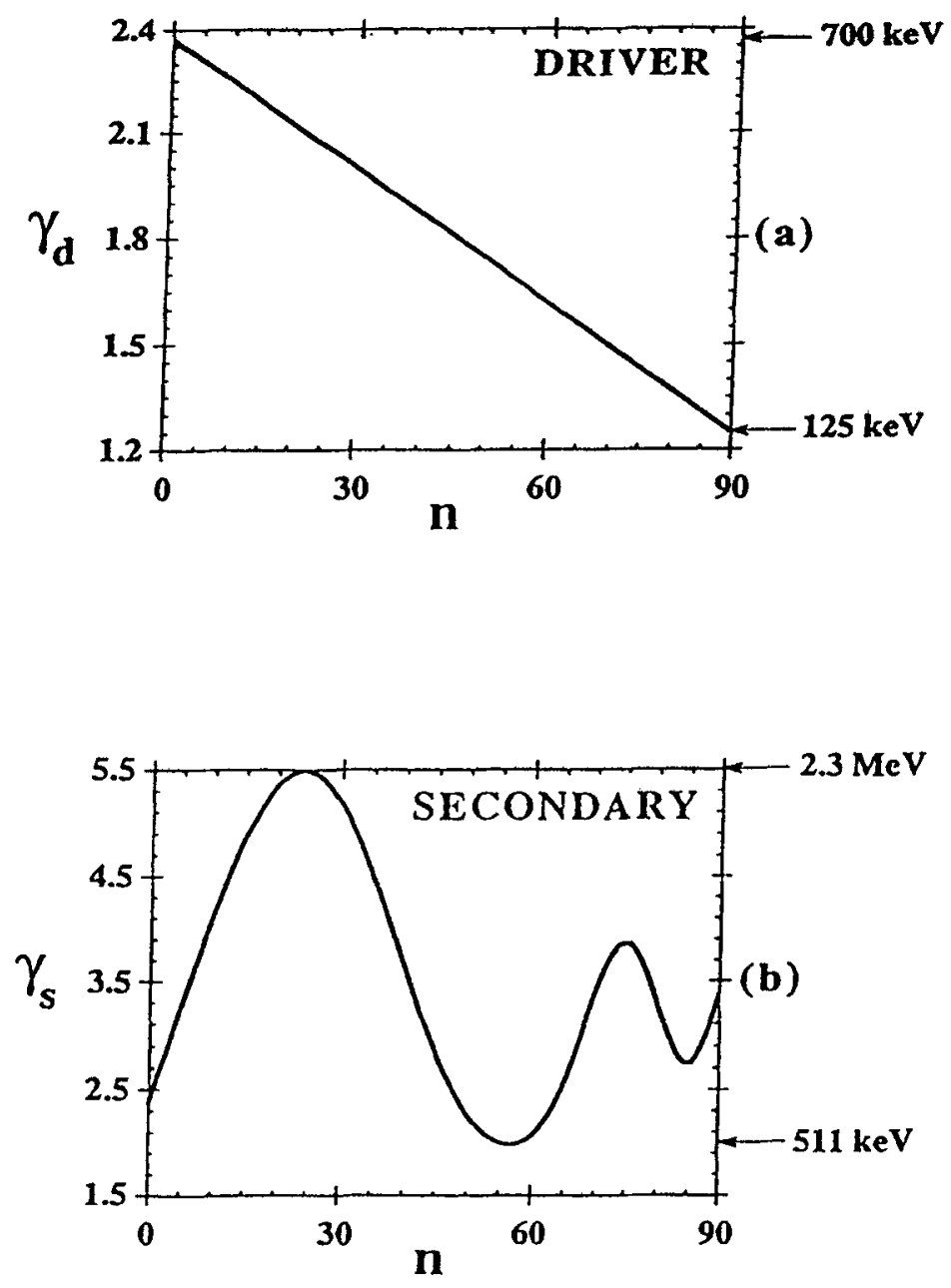

Figure 3. Evolution of the relativistic mass factors when the driver beam radius $r_{0}$ is a constant: (a) the driver beam, (b) the secondary beam. Phase slippage prohibits continual acceleration of the secondary beam.

The phase slippage may be corrected [4] by adjusting the primary beam's radius $r_{0}$. Consider, for example, the worst case of phase slippage where the 
primary beam and the secondary beam arrive at a cavity $180^{\circ}$ out of phase, as shown in Fig. $2 b$. If the primary beam's radius $r_{0}$ is less than a, it generates an $\mathrm{rf}$ electric field which would retard both beams during the time when the primary beam occupies the cavity. However, when the charge bunch of the primary beam resides in the cavity, there are few particles in the secondary beam residing in the same cavity because both beams arrive at the cavity $180^{\circ}$ out of phase. By the time the charge bunch of the primary beam is about to leave the cavity, the rf electric field is about to change sign, at which time the charge bunch of the secondary beam is about to enter the cavity, whose rf electric field then begins to accelerate the entering bunch on the secondary beam. Thus, the phase slippage problem can be corrected by a simple cure: At the locations where the bunches of both beams enter the cavity with the same phase, place $r_{O}$ outside $a$. When the bunches of both beams arrive at the cavity $180^{\circ}$ out of phase, place $r_{0}$ inside a.

Mathematically, it is easy to see from Eqs. (3) and (4) that $\gamma_{S}$ is a monotonically increasing function of $\mathrm{n}$ if $\mathrm{r}_{\mathrm{O}}$ is tapered in such a way that $\left(r_{O}-a\right) \cos \left(\theta_{S}-\theta_{d}\right) \geq 0$. Thus, if we write the primary beam radius $r_{O}$ as

$$
\mathrm{r}_{\mathrm{o}}-\mathrm{a}=\Delta \cos (\psi),
$$

where $\Delta$ is the amplitude and $\psi$ is the phase of the modulation in $r_{0}$, the general phase focusing condition reads

$$
\mathrm{d} \psi / \mathrm{dn}=\mathrm{d}\left(\theta_{\mathrm{S}}-\theta_{\mathrm{d}}\right) / \mathrm{dn} .
$$

Including this modification, with $\Delta=3.322 \mathrm{~cm}-3.146 \mathrm{~cm}=0.176 \mathrm{~cm}$, and keeping all other parameters the same as in Fig. 3, we obtain Fig. 4. In Fig. 4, we see that the primary beam's energy monotonically decreases from $700 \mathrm{keV}$ to 415 $\mathrm{keV}$ over $90 \mathrm{~cm}$, whereas the secondary beam's energy increases monotonically from $700 \mathrm{keV}$ to a maximum of $4.8 \mathrm{MeV}$ over the same distance, in sharp contrast to Fig. 3b. The loss of $285 \mathrm{keV}$ in the primary beam and the gain of $4.1 \mathrm{MeV}$ in the secondary beam implies an effective transformer ratio of about $4.1 \mathrm{MeV} / 285$ $\mathrm{keV}=14.4$.

The modulation in the annular beam radius may be readily achieved by a proper adjustment of the external solenoidal magnetic field which is often used for beam focusing and beam transport. Since the rate of change of energy depends on the annular beam radius $r_{O}$ [cf. Eqs. (1), (3)], the output energy of the accelerated beam may also be controlled by the same external magnetic field coils.

The above ideas may be tested in a proof-of-principle experiment with parameters similar to those used to produce Fig. 4. The primary beam may be obtained, for example, from the Michigan Electron Long-Beam Accelerator (MELBA [5]), which operates with diode parameters of $700 \mathrm{keV}$, current up to 10 
$\mathrm{kA}$, and flattop pulse length up to $1 \mu \mathrm{s}$. This primary beam may be modulated using the proven techniques by Friedman et al. [2]. Note that the average acceleration gradient of $45 \mathrm{kV} / \mathrm{cm}$ and the peak acceleration gradient of about 80 $\mathrm{kV} / \mathrm{cm}$ implied by Fig. 4 are well within the rf breakdown limit. If we assume an acceleration efficiency of 25 per cent, a secondary beam of more than 8 amps of current may be accelerated to $5 \mathrm{MeV}$ in less than a meter in this proof-of-principle experiment.
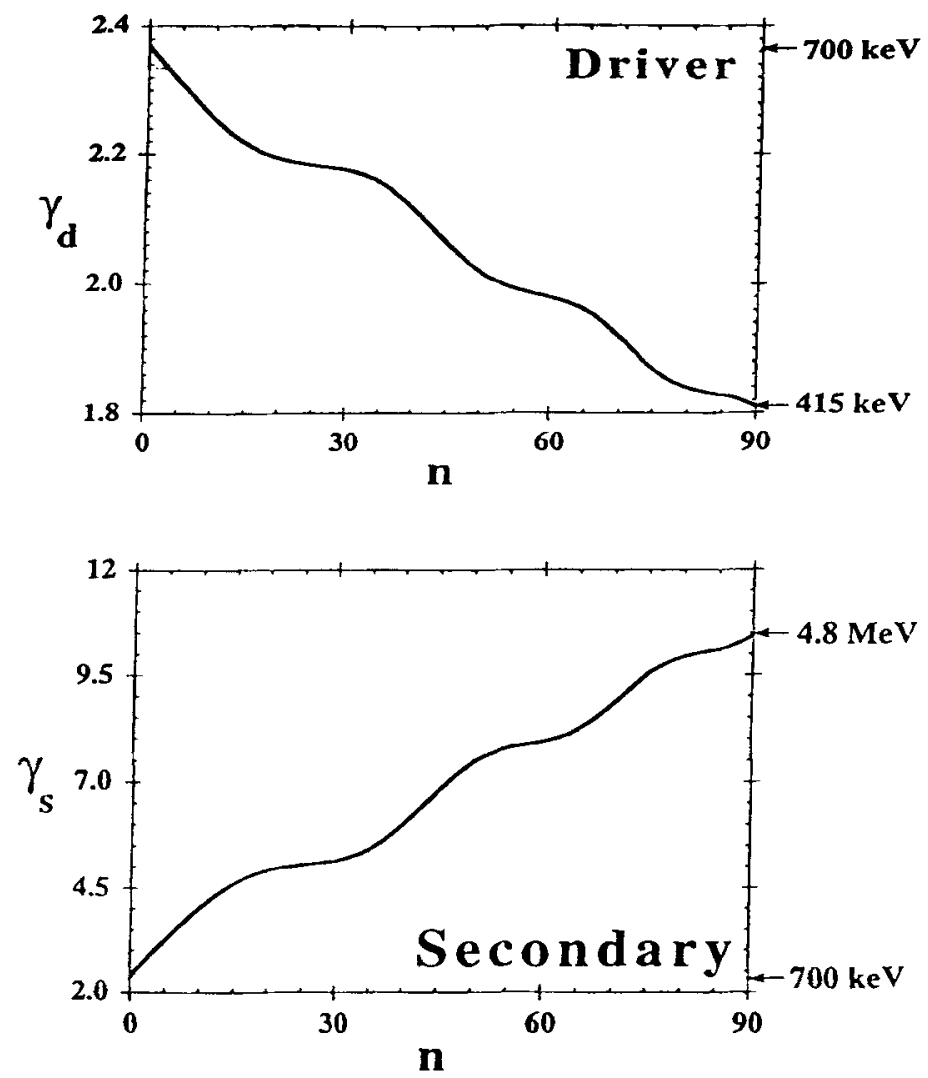

Figure 4. Evolution of the relativistic mass factors when the driver beam radius $r_{o}$ is modulated to compensate phase slippage: (a) the driver beam, (b) the secondary beam. 


\section{ISSUES}

We shall address some of the issues which may affect the eventual usefulness of the twobetron. They concern the scaling laws, beam breakup instabilities, wakefield effects, effects of finite beam thickness, coupling among cavities, modification of $\mathrm{rf}$ characteristic by the intense driver beam, and the integrity of the primary beam modulation in the accelerating structure.

\section{Scaling}

The average energy gain per cavity by the secondary beam in the twobetron is

$$
<\mathrm{E}_{\mathrm{s}}>=(16.3 \mathrm{keV}) \times \mathrm{Q} \times\left(\mathrm{I}_{\mathrm{d}} / 1 \mathrm{kA}\right) \times(\Delta / \mathrm{a}) .
$$

The transformer ratio, $\mathrm{R}$, which is the ratio of the energy gain in the secondary beam to the energy loss in the primary beam, is

$$
\mathrm{R}=0.803(\mathrm{a} / \Delta)
$$

The maximum amount of secondary beam current, $I_{s}$, that can be accelerated is limited to

$$
I_{s}<\frac{I_{d}}{2 R}
$$

Given a driver beam current, we cannot make the acceleration gradient (i.e., $\left\langle\mathrm{E}_{\mathrm{s}}\right\rangle$ ) excessively high by using a very high $\mathrm{Q}$ cavity. A practical limit on $\left\langle\mathrm{E}_{\mathrm{s}}\right\rangle$ is set by rf breakdown in the cavities [6].

In general, a transformer ratio $\mathrm{R}$ of the order of 10 seems achievable. A twostage twobetron, in which the accelerated beam of the first stage is used as the driver beam in the second stage, will provide voltage multiplication by a factor of 100, while the output current is correspondingly much reduced [cf. Eq. 10].

\section{Primary Beam Instabilties}

The intense driver beam passing through a sequence of cavities is highly vulnerable to beam breakup instabilities (BBU [7-10]). However, we have recently found that $\mathrm{BBU}$ in an annular beam may be far less serious than a pencil beam [8]. Specifically, in the absence of other stabilizing mechanisms such as stagger tune and betatron frequency spread, the number of e-folds, $(N$, in BBU amplitude growth) over an accelerator of length $\mathrm{z}$ is given by [9] 


$$
N=\left(\frac{\omega_{b} z}{\beta_{d} c}\right)\left(\frac{\omega_{b}}{\omega_{\beta}}\right) \varepsilon Q_{b},
$$

where $\omega_{b}$ is the frequency of the deflecting mode with quality factor $\mathrm{Q}_{b}, \omega_{\beta}$ is the betatron frequency associated with the focusing field, $\varepsilon=0.0041\left(\beta_{d} / \gamma_{d}\right)\left(I_{d} / 1 k A\right)$ is the dimensionless coupling constant, and $\beta_{\mathrm{d}}, \gamma_{\mathrm{d}}$ are defined in Eq. 5. For a solenoidal magnetic field of $10 \mathrm{kG}$ and a dipole mode $\mathrm{Qb}_{\mathrm{b}}$ of $100, N=1.8$ for a $500 \mathrm{~ns}, 0.5 \mathrm{kA}$ driver beam in a $90 \mathrm{~cm}$ accelerator structure, as in the numerical example.

We conjecture that the longitudinal (Robinson-like) instability [10] probably is not important for the twobetron, at least in the proposed proof-of-principle experiment. Unlike a circular accelerator, the present scheme is single-pass. Its acceleration length is quite short, its length is only slightly over one wavelength in the radius modulation. Moreover, the drive frequency may be adjusted to be on the "right side" of the structure frequency to avoid the Robinson-like instability.

In a preliminary particle simulation, we find that the current modulation is preserved on the primary beam, after it is made to propagate through the accelerating structure, using the beam and structure parameters that are being planned for a proof-of-principle experiment.

\section{Wakefield Effects}

We have also examined the effects of the transverse wake on the driver beam, and found that a nominal value of solenoidal field $B_{O}=10 \mathrm{kG}$ would render the effects of the transverse wakefield on the driver beam unimportant. Specifically, under the condition $\Omega \gg \omega / \gamma_{d}\left(1+\beta_{d}\right)$, where $\Omega$ is the nonrelativistic cyclotron frequency associated with $B_{o}$ and the other symbols are the same as in Eq. (5), the electron motion is adiabatic along the composite (DC + rf) magnetic field line. The maximum angular displacement, from the mean, is estimated to be

$$
I_{\theta}=0.52(c / \omega)\left(E_{a} / c B_{o}\right) \beta_{d} /\left(1-\beta_{d}\right)
$$

where $\mathrm{E}_{\mathrm{a}}$ is the maximum accelerating electric field experienced by the secondary beam. The maximum radial displacement is

$$
I_{\mathrm{r}}=I_{\theta} \lambda_{\mathrm{L}} / \lambda_{\mathrm{m}}
$$

where $\lambda_{L}=2 \pi \beta_{d} \gamma_{d} c / \Omega$ and $\lambda_{m}$ is the axial wavelength associated with the modulation in the driver beam radius. For the parameters used in the numerical example, $I_{\theta} \leq 0.2 \mathrm{~cm}$, and $I_{T} \leq 0.0058 \mathrm{~cm}$. The spread in momentum, $d p$, in the 
driver beam may introduce a variation in its annular beam radius, $\mathrm{dr}_{\mathrm{o}}$. It is estimated that

$$
\mathrm{dr}_{\mathrm{o}} \leq\left[2\left(\lambda_{\mathrm{L}}^{2} / \lambda_{\mathrm{m}}^{2}\right) \Delta+0.083 \lambda_{\mathrm{L}} \mathrm{E}_{\mathrm{a}} / \mathrm{cB}_{\mathrm{o}}\right] \mathrm{dp} / \mathrm{p}
$$

where $\Delta$ is the amplitude of the modulation in the driver beam radius. Using the parameters in the numerical example [Fig. 4], we find $\mathrm{dr}_{\mathrm{o}}<0.0061 \mathrm{~cm}$ if $\mathrm{dp} / \mathrm{p}<$ 1. Thus, the effectiveness of radius modulation is not affected by momentum spread.

\section{RF Coupling Between Cavities}

We have for simplicity assumed that the cavities are isolated from one another electromagnetically when the beam is absent. There are several ways to reduce the coupling among neighboring cavities. The inductive coupling at the annular slots, through which the driver beam passes, may be cancelled by the capacitive coupling at the center hole, and if necessary, by introducing additional holes near the rf electric field maximum (so as to increase the capacitive coupling) that is close to the outer wall of the cavity [Fig. 1]. Alternatively, conducting wires may be inserted radially across the annular gap to reduce the inductive coupling. Multiple pencil beams may also be used as the driver. These pencil beams pass through holes that are distributed annularly. In the event that the neighboring cavities are not completely isolated electromagnetically, a traveling wave formulation would be required; but the radius modulation that is proposed in this paper still provides an external control to ensure phase focusing.

The presence of intense space charge in the driver beam complicates matters substantially, as it is known to modify the rf characteristics in an unpredictable manner. Such modifications include a detune of the structure frequency and modification of the gap transit-time factor, especially if a virtual cathode is on the verge of being formed [2, 11]. Other modes may be excited. Indeed, mode competition is a major area that requires considerable attention in the twobetron concept.

\section{Effect of Finite Thickness in the Driver Beam}

The effect of finite thickness, $\tau$, in the driver beam is found to be much less important than its mean radius, $r_{0}$. The finite beam thickness modifies Eq. 3 to read

$$
\delta=-1.249\left(\mathrm{r}_{\mathrm{o}}-\mathrm{a}\right) / \mathrm{a}-0.05(\tau / \mathrm{a})^{2}
$$

The last term in Eq. 15 is usually much smaller than the first term even if $\tau$ and the radius modulation amplitude, $\Delta$, are of the same order of magnitude (Eq. 6 ). 


\section{CONCLUDING REMARKS}

In summary, the twobetron has the potential of converting many existing pulse power systems into compact rf accelerators that are suitable for industrial and medical applications. The driver beam is a modulated electron beam of annular shape and low energy. The secondary beam is an on-axis pencil beam. The secondary beam may reach an energy up to $10 \mathrm{MeV}$ in one to two meters. Transformer ratio on the order of ten is considered feasible for each stage. Phase focusing and energy tunability of the accelerated beam may be provided by an external magnetic field, which controls the radius of the primary beam.

Excitation of the undesirable modes by the driver beam is perhaps the single most important issue in the twobetron concept.

\section{ACKNOWLEDGMENTS}

We gratefully acknowledge Perry Wilson for pointing out an error of a factor of two in the numerical coefficient in Eq. (2) in the original manuscript. This work was supported by SDIO-BMD/IST/ONR.

\section{REFERENCES}

1. G. Voss and T. Weiland, DESY Reports \#M82-10 (1982) and M82-079 (1982); A. M. Sessler and S. S. Yu, Phys. Rev. Lett. 58, 2439 (1987); M. A. Allen et al., ibid., 63, 2472 (1989); W. Gai et al., ibid., 61, 2765 (1988); H. Figueroa et al., ibid., 60, 2144 (1990); J. B. Rosenzweig et al., Phys. Fluids B2, 1376 (1990); A. M. Sessler et al., Part. Accel. 31, 1277 (1990), Nuclear Instrum Meth. A306, 592 (1991); D. B. Hopkins et al., ibid., 228, 15 (1984); D. H. Whittum et al., Phys. Rev. A43, 294 (1991); Also, in AIP Conf. Proc. no. 279, Ed. J. Wurtele (1993).

2. M. Friedman and V. Serlin, Phys. Rev. Lett. 55, 2860 (1985); M. Friedman et al., J. Appl. Phys. 64, 3353 (1988); J. Krall and Y. Y. Lau, Appl. Phys. Lett. 52, 431 (1988); Y. Y. Lau et al., IEEE Trans. Plasma Sciences PS-18, 553 (1990).

3. M. Friedman et al., Phys. Rev. Lett. 63, 2468 (1989); Rev. Sci. Instrum. 61,171 (1990); Also, see Proceedings of SPIE Meetings, no. 1407 (1991), no. 1629 (1992), no. 1872 (1993); Ed. H. E. Brandt.

4. Ya. S. Derbenev, Y. Y. Lau, and R. M. Gilgenbach, Phys. Rev. Lett. 72, 3025 (1994).

5. R. M. Gilgenbach et al., in Digest of Fifth IEEE Pulse Power Conference (IEEE, New York, 1985), p. 126.

6. G. A. Loew and J. W. Wang, SLAC Publication No. 4647 (May, 1988).

7. W. K. H. Panofsky and M. Bander, Rev. Sci. Instrum. 39, 206 (1968). A recent experimental study of beam breakup in high current beams is reported by P. R. Menge et al., J. Appl. Phys. 75, 1258 (1994). 
8. Y. Y. Lau and J. W. Luginsland, J. Appl. Phys. 74, 5877 (1993). In the line above Eq. (7) of this paper, the factor $(\mathrm{I} / 1 \mathrm{kA})$ should read $(\mathrm{I} / 17 \mathrm{kA})$.

9. V. K. Neil, L. S. Hall, and R. K. Cooper, Part. Accel. 9, 213 (1979); P. B. Wilson, AIP Conf. Proc. No. 87, p. 452 (1982).

10. A. W. Chao, Physics of Collective Beam Instabilities in High Energy Accelerators, (Wiley, New York, 1993).

11. D. G. Colombant and Y. Y. Lau, Phys. Rev. Lett. 64, 2320 (1990); Phys. Rev. A45, R2179 (1992). 